\title{
Dynamics of Race Structures of Pyricularia oryzae Populations Across 18 Seasons in Guangdong Province, China
}

\author{
Zhipeng Huang, ${ }^{1}$ Jinyan Wang, ${ }^{1}$ Yaling Zhang, ${ }^{1,2}$ Yongxiang Yao, ${ }^{1,3}$ Lifei Huang, ${ }^{1,4}$ Xueyan Yang, ${ }^{1,5}$ Ling Wang, \\ and Qinghua Pan ${ }^{1, \dagger}$ \\ ${ }^{1}$ State Key Laboratory for Conservation and Utilization of Subtropic Agrobioresources, Guangdong Provincial Key Laboratory \\ of Plant Molecular Breeding, Guangdong Laboratory for Lingnan Modern Agriculture, College of Agriculture, South China \\ Agricultural University, Guangzhou 510642, China \\ ${ }^{2}$ College of Agronomy, Heilongjiang Bayi Agricultural University, Daqing, Heilongjiang 163319, China \\ ${ }^{3}$ Dandong Academy of Agricultural Sciences, Dandong, Liaoning 118109, China \\ ${ }^{4}$ Crops Research Institute, Guangdong Provincial Key Laboratory of Crops Genetics and Improvement, Guangdong Academy of \\ Agricultural Sciences, Guangzhou, Guangdong 510640, China \\ ${ }^{5}$ Linyi University, Linyi, Shandong 276000, China
}

\begin{abstract}
Rice blast, caused by Pyricularia oryzae, is one of the most damaging fungal diseases affecting rice. Understanding how the pathogen's race structure varies over time supports the efforts of rice breeders to develop improved cultivars. Here, the race structure of $P$. oryzae in Guangdong province, China, where rice is cropped twice per year, was assessed over 18 seasons from 1999 through 2008. The analysis was based on the reactions of a panel of seven differential Chinese cultivars to inoculation with a set of 1,248 isolates of $P$. oryzae in the province. The "total race frequency" parameter ranged from 14.7 to $39.7 \%$, and the "race diversity index" ranged from 0.63 to 0.93 . Twelve (ZA63, ZA31, ZA29, ZA21, ZA13, ZA9, ZB30, ZB17, ZB8, ZB2, ZC14, and ZC8) and two (ZD8

and nine indica type races (ZB1, ZB5, ZB6, ZB7, ZB13, ZB15, ZC5, $\mathrm{ZC13}$, and $\mathrm{ZC} 15$ ) and one japonica type race (ZG1) were deemed to be population-dominant; the "total top two race isolate frequency" parameter ranged from 29.8 to $74.5 \%$. On the host side, dynamics of resistance structures of the differential set were divided into three patterns: Both Tetep and Kanto 51 expressed the highest and most stable resistance, both Sifeng 43 and Lijiangxintuanheigu conveyed much lower and unstable resistance, and Zhenlong 13, Dongnong 363, and Heijiang 18 performed intermediate and seasonally dynamic resistance. Three interesting points distinguishing race structures of $P$. oryzae populations in southern and northeastern China were also discussed.
\end{abstract} and $Z \mathrm{D} 3$ ) races were recognized as specific to indica and japonica rice types, respectively. Of the 59 distinct races identified, only two indica type races (ZC13 and $\mathrm{ZC} 15)$ were identified as population-common,
Keywords: cereals and grains, disease development and spread, epidemiology, field crops, fungi
Rice blast, caused by the fungus Pyricularia oryzae Cavara (syn. Magnaporthe oryzae B.C. Couch), is a highly destructive disease, most reliably controlled by the deployment of host resistance genes (Couch and Kohn 2002; Nguyet et al. 2020; Pan et al. 1996; Wang et al. 2017). For a successful resistance breeding program, it is essential to understand how the causative pathogen's race structure varies over time and space and to be familiar with the resistance genes in current cultivars and elite breeding lines (Lei et al. 2011; Wang et al. 2017; Zhang et al. 2017, 2019).

Sets of differential cultivars, the members of which have been chosen to harbor all of the regionally important host resistance genes, have been developed as an effective means of tracking the dynamics of pathogen populations in a number of host-pathogen systems

${ }^{\dagger}$ Corresponding author: Q. Pan; panqh@ scau.edu.cn

Z. Huang, J. Wang, and Y. Zhang contributed equally to this work.

Funding: This study received funding from the National Key R\&D Project (2016YDF0100601), the National Transgenic Research Project (2016ZX08001002), the National Natural Science Foundation of China (31870137; U1131003), the Guangdong Provincial Key Laboratory of Plant Molecular Breeding (GPKLPMB201801), and the Guangzhou Key R\&D Project (201504010029).

*The $\boldsymbol{e}$-Xtra logo stands for "electronic extra" and indicates there are supplementary tables published online.

The author(s) declare no conflict of interest.

Accepted for publication 21 July 2020.

Copyright $\odot 2021$ The Authors. This is an open access article distributed under the CC BY-NC-ND 4.0 International license.
(Atkins et al. 1967; Feng et al. 2018; Fernando et al. 2018; Liu et al. 2017; Wang et al. 2017; Zhang et al. 2017, 2019). The Chinese differential cultivar (CDC) panel, which was based on the National Collaboration Initiative for the rice blast pathosystem, is still the most reliable set for creating the easy-to-use information on both race and resistance structures, because the set includes representatives of both indica and japonica type cultivars, among which all of the major host resistance genes used in Chinese rice breeding programs are represented (All China Corporation of Research on Physiological Races of Pyricularia oryzae 1980; Zhang et al. 2017, 2019). Since its inception in 1980, the CDCs have been used to define the pathogen's race structure in a number of rice-growing regions of the country (Gao et al. 2017; Huo et al. 1980; Lai et al. 1998; Li and Zheng 1989; Pan et al. 1998; Shang et al. 1996; Shi et al. 2015; Xing et al. 2017; Yang et al. 1995). However, there was little information about long-term management of the disease, because of the lack of a series of historical populations and a suitable method being used to dissect the race structures for application (Zhang et al. 2017, 2019).

Rice is one of the top three cereal crops in China in terms of area (rice, wheat, and maize covered 30.73, 27.86, and 22.76 million hectares, respectively, per year from 1986 to 2005) and production (rice, maize, and wheat produced $182.36,106.58$, and 98.74 million tons, respectively, per year from 1986 to 2005) (Cheng 2010). Based on the Chinese administrative areas in combination with their topological, climatic, and edaphic characters, six rice cropping zones (RCZs) have been defined, namely southern (RCZ I), central and eastern (RCZ II; currently called the middle and lower reaches of the Yangtze River), southwestern (RCZ III), northern (RCZ IV), northeastern (RCZ V), and northwestern (RCZ VI) zones (Cheng 2010; Mei et al. 1988; Zhang et al. 2017). The climate of Guangdong province (GD; 20.09-25.31 ${ }^{\circ}$ north), lying within RCZ I, is characterized by high ambient temperatures and high humidity, allowing rice to be 
generally cropped twice per year. The first cropping season (locally called the earlier season) lasts from March to July, and the second (the later one) lasts from July to December, and most of the cultivars grown in GD are indica types (Wu et al. 2004; Xu 1988; Zhang et al. 2017). Rice blast is a significant constraint to production in GD (Huo et al. 1980; Lai et al. 1998; Pan et al. 1998; Yang et al. 1995; Zhang et al. 2017). Whereas an earlier study used the CDCs to document differences between the race structures of $P$. oryzae in GD and the rest of China (Zhang et al. 2017), the focus of the present study was to characterize the dynamics of the GD population over an extended period (1999 to 2008).

\section{Materials and Methods}

Pathogen isolates and inoculum preparation. Rice panicles showing symptoms of rice blast were sampled across GD in 18 consecutive seasons, starting from the later season of 1999 (Pop-99L) and ending after the earlier season of 2008 (Pop-08E) (Supplementary Table S1). In each case, a monoconidial isolate was developed from each sample and stored on dry filter paper at $-20^{\circ} \mathrm{C}$, according to an established protocol (Li et al. 2018; Zhang et al. 2017, 2019). The resulting collection comprised 47 to 97 isolates per population, which were selected based on information on both locations and host cultivars, representing an overall collection of 1,248 isolates (Supplementary Table S1). Standard methods were used to prepare inoculum from the isolates on a rice bran medium ( $20 \mathrm{~g} /$ liter of rice bran, 2 $\mathrm{g} /$ liter of yeast extract, and $11 \mathrm{~g} /$ liter of agar) (Pan et al. 1996, 2003a). From each culture, an aqueous conidial suspension of $\geq 10^{5} / \mathrm{ml}$ was used to inoculate the CDC panel.

CDC panel and the coding of pathogen races. The CDC panel comprised three indica type (Tetep, Zhenlong 13, and Sifeng 43) and four japonica type (Dongnong 363, Kanto 51, Hejiang 18, and Lijiangxintuanheigu [LTH]) cultivars (Supplementary Table S2; All China Corporation of Research on Physiological Races of Pyricularia oryzae 1980; Zhang et al. 2017, 2019). Seedlings were inoculated at the four- to six-leaf stage, having been raised in $58-\times 38-\times$ $8-\mathrm{cm}$ trays in a greenhouse where the temperature was maintained between 20 and $35^{\circ} \mathrm{C}$ (Pan et al. 2003a). After inoculation, the plants were first held for $24 \mathrm{~h}$ in a dark chamber providing a relative humidity of $95 \%$ and a temperature of 25 to $28^{\circ} \mathrm{C}$, then transferred into a plastic tunnel held at 25 to $30^{\circ} \mathrm{C}$ to allow the disease to develop (Pan et al. 2003a). The host reaction was rated 6 days postinoculation according to the Pan et al. (1996) scale (0 to 3, resistant; 4 to 5, susceptible). The reaction of each host-pathogen combination was validated by carrying out two independent inoculations per isolate; where the two replicates produced an inconsistent outcome, the higher score was used (Pan et al. 1996, 2003a; Xing et al. 2017; Zhang et al. 2017, 2019).

Each CDC member was assigned an alphanumeric code (A64, Tetep; B32, Zhenlong 13; C16, Sifeng 43; D8, Dongnong 363; E4, Kanto 51; F2, Hejiang 18; and G1, LTH), based on their overall profile of resistance against a national set of 1,739 P. oryzae isolates (All China Corporation of Research on Physiological Races of Pyricularia oryzae 1980). Each isolate was also assigned a code, based on all 128 possible reactions (phenotypes) on the seven CDCs $\left(2^{7}=128\right)$. Races are classified into groups A through C (derived from indica $\mathrm{CDC}$ entries) and $\mathrm{D}$ through $\mathrm{G}$ (derived from $\mathrm{ja}$ ponica $\mathrm{CDC}$ entries), and therefore they were also called indica and japonica type races, respectively. A special race group $\mathrm{H}$, which was given to isolates that were incompatible with all seven CDC entries, was also called japonica type race (see details in Supplementary Table S2; All China Corporation of Research on Physiological Races of Pyricularia oryzae 1980; Zhang et al. 2017, 2019).

Data analysis. The race structure of each population was assessed by four criteria (race diversity, specific race structure, common race structure, and dominant race structure) and quantified by 10 parameters (details provided in Supplementary Table S3; also see Zhang et al. 2017, 2019). On the host side, resistance structure was characterized by two parameters, namely resistance frequency and total resistance frequency. A $\chi^{2}$ test was performed to determine the homogeneity of parameters related, if applicable (Supplementary Table S3; Fernando et al. 2018; Zhang et al. 2019).

\section{Results}

Race diversity. The number of races identified in a single population varied from 10 (Pop-99L and Pop-00E) to 27 (Pop-07E); the range in the total race frequency $\left(f_{t r}\right)$ was 14.7 (Pop-02L) to $39.7 \%$ (Pop-07E) (Table 1; Supplementary Tables S1, S3, S4). Based on the number of the isolates represented in a given population, the race diversity index $\left(h_{r d i}\right)$ ranged from 0.63 (Pop-05L) to 0.93 (Pop-01L). The Margalef index $\left(m_{i}\right)$ of race richness ranged from 2.21 (Pop-00E) to 6.16 (Pop-07E). A homogeneity test for $f_{t r}, h_{r d i}$, and $m_{i}$ revealed that only $f_{t r}$ identified significant difference among all the populations tested (Table 1).

Specific race structure. In seven of the 18 populations, there were 12 indica type (ZA63, ZA31, ZA29, ZA21, ZA13, ZA9, ZB30, $\mathrm{ZB} 17, \mathrm{ZB} 8$, ZB2, ZC14, and ZC8) and two japonica type (ZD8 and ZD3) races recognized as population-specific (Supplementary Table S2). Five of the seven populations were the earlier seasonderived ones. The number of population-specific races in a given population ( $p s r$ ) ranged from one to four, resulting in estimates for the population-specific race frequency $\left(f_{p s r}\right)$ of 4.8 (Pop-03E) to $16.0 \%$ (Pop-08E) (Tables 1 and 2). The numbers of isolates involved ranged from one to seven, giving rise to estimates of the total population-specific race isolate frequencies $\left(f_{t p s r i}\right)$ of 1.3 (Pop-03E) to $10.3 \%$ (Pop-07E). A homogeneity test conducted on $f_{p s r}$ and $f_{t p s r i}$ revealed significant differences between the seven populations for the latter parameter but not for the former one.

Common race structure. Of 59 identified races, only two indica type races (ZC13 and $\mathrm{ZC15}$ ) were found in all 18 populations (Table 3; Supplementary Table S1). The population common race isolate frequency for the first one, $\mathrm{ZC} 13\left(f_{\text {pcri-1 }}\right)$, ranged from 1.7 (Pop-00E) to $24.0 \%$ (Pop-02L), and that for the second one, ZC15 $\left(f_{\text {pcri-2 }}\right.$ ), from 1.3 (Pop-04E, Pop-08E) to $34.0 \%$ (Pop-00L). The total population common race isolate frequency $\left(f_{t p c r i}\right)$ ranged from 7.4 (Pop-07E) to $51.5 \%$ (Pop-00L) and the population common race frequency $\left(f_{p c r}\right)$ from 7.4 (Pop-07E) to $20.0 \%$ (Pop-99L, Pop-00E). Applying the homogeneity test revealed significant differences for $f_{\text {pcri-1 }}, f_{\text {pcri-2 } 2}$, and $f_{\text {tpcri }}$.

Dominant race structure. Nine indica type races (ZB1, ZB5, ZB6, ZB7, ZB13, ZB15, ZC5, ZC13, and ZC15) and one japonica type race ( $Z G 1)$ were identified as dominant across the 18 populations (Table 4; Supplementary Table S4). The isolate frequency for the first race $\left(f_{t 2 r i-1}\right)$ ranged from 60.3 (Pop-05E) to $19.1 \%$ (Pop$01 \mathrm{~L}$ ), and that for the second one $\left(f_{t 2 r i-2}\right)$ ranged from 24.6 (Pop05L) to $6.9 \%$ (Pop-05E); together, these two races generated a total top two race isolate frequency $\left(f_{t t 2 r i}\right)$ of 29.8 (Pop-01L) to $74.5 \%$ (Pop-99L). Each of $f_{t 2 r i-1}, f_{t 2 r i-2}$, and $f_{t t 2 r i}$ varied significantly between the 18 populations.

Resistance structure. There were three dynamic types of resistance among seven CDCs when challenged by all isolates in the 18 P. oryzae populations over the period (Fig. 1; Supplementary Table S4). Both Tetep and Kanto 51 expressed a higher and more stable level of resistance over the period as a whole. In contrast, Sifeng 43 and LTH exhibited an inferior and more unstable level. The performance of the other three differentials, Zhenlong 13, Dongnong 363 , and Heijiang 18, was intermediate and seasonally dynamic.

\section{Discussion}

Because an understanding of race structure of a pathogen population supports a rational deployment of host resistance cultivars, the literature includes a number of studies directed to this goal. However, there was less information that could be directly addressed to the major concerns from plant breeders and producers (Liu et al. 2017; Wang et al. 2017; Zhang et al. 2017, 2019). In the current study, dynamics of race structures of $P$. oryzae over the 18 seasons were dissected into four aspects (race diversity, specific race structure, common race structure, and dominant race structure) with 10 comparable parameters (Tables 1, 2, 3, and 4; Supplementary Table S3). A substantial level of race diversity was detected, as evidenced by the 
range in $f_{t r}(14.7$ to $39.7 \%$ ) (Table 1$)$, which was greater than what was deduced from a 10-year study of $P$. oryzae populations in Hei-

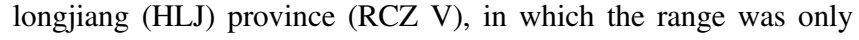
12.4 to $26.0 \%$ (Zhang et al. 2019). The race diversity of $P$. oryzae populations in the RCZ I was higher than that in the RCZ V of China (Lai et al. 1998; Li and Zheng 1989; Shang et al. 1996; Wu et al. 2004; Zhang et al. 2017, 2019). Part of the basis for this geographic difference in race diversity probably lies in the nature of the two ecosystems: in RCZ I, rice is cropped twice per year, whereas in RCZ V, the climate supports only a single cropping season (middle season, from May to October) (Cheng 2010; Zhang et al. 2017, 2019). Intriguingly, there appears to be a difference in the timing of when race diversity jumped: In GD, the critical year was 2007 (Table 2), whereas in HLJ it was 2011 (Zhang et al. 2019). The likely basis for this time lag is that breeding for resistance began earlier in GD than in HLJ (Zhang et al. 2017, 2019), given that the appearance of new pathogen races has coincided with changes in breeding strategy in a number of other pathosystems, notably wheat-Puccinia striiformis in the United States since 2000 (Liu et al. 2017), canola-Leptosphaeria maculans in Canada since 2012 (Fernando et al. 2018), maize-Exserohilum turcicum in Canada since 2014 (Jindal et al. 2019), soybean-Phytophthora sojae in the United States since 2013 (Dorrance et al. 2016), and spinach-Peronospora effusa in the United States since 1990 (Feng et al. 2018). The major determinant in these situations appears to have been host-driven selection, reflecting the deployment of a greater diversity of cultivars (Feng et al. 2018; Fernando et al. 2018; Jindal et al. 2019; Liu et al. 2017; Wang et al. 2017; Wu et al. 2004; Zhang et al. 2017, 2019).

Improved information on race structure, particularly the common and dominant races, can direct the producers' choice of cultivars (Correa-Victoria and Zeigler 1993; Fernando et al. 2018; PaczosGrzęda and Sowa 2019; Yang et al. 1995; Zhang et al. 2017, 2019). Of the $59 P$. oryzae races identified, only two indica type ones

Table 1. Diversity shown by the 18 Pyricularia oryzae populations collected from Guangdong province over the period 1999-2008, assessed by challenging a set of Chinese differential cultivars

\begin{tabular}{|c|c|c|c|c|}
\hline $\begin{array}{l}\text { Population (no. } \\
\text { of isolates) }^{\mathrm{a}}\end{array}$ & $\begin{array}{c}\text { No. of } \\
\text { races }(t r)\end{array}$ & $\begin{array}{c}\text { Race } \\
\text { frequency } \\
\left(f_{t r}\right)\end{array}$ & $\begin{array}{c}\text { Race diversity } \\
\text { index }\left(h_{r d i}\right)^{\mathbf{b}}\end{array}$ & $\begin{array}{r}\text { Margalef } \\
\text { index }\left(m_{i}\right)^{\mathrm{c}}\end{array}$ \\
\hline 99L (51) & 10 & 19.6 & 0.64 & 2.29 \\
\hline O0E (59) & 10 & 16.9 & 0.82 & 2.21 \\
\hline 00L (97) & 17 & 17.5 & 0.83 & 3.50 \\
\hline 01E (57) & 11 & 19.3 & 0.84 & 2.47 \\
\hline 01L (47) & 17 & 36.2 & 0.93 & 4.16 \\
\hline 02E (85) & 15 & 17.6 & 0.90 & 3.15 \\
\hline 02L (75) & 11 & 14.7 & 0.85 & 2.32 \\
\hline 03E (80) & 21 & 26.3 & 0.91 & 4.56 \\
\hline 03L (81) & 18 & 22.2 & 0.89 & 3.87 \\
\hline 04E (78) & 16 & 20.5 & 0.70 & 3.44 \\
\hline 04L (78) & 19 & 24.4 & 0.91 & 4.13 \\
\hline 05E (58) & 13 & 22.4 & 0.80 & 2.96 \\
\hline $05 \mathrm{~L}(57)$ & 14 & 24.6 & 0.63 & 3.22 \\
\hline $06 \mathrm{E}(60)$ & 14 & 23.3 & 0.79 & 3.18 \\
\hline $06 \mathrm{~L}(60)$ & 11 & 18.3 & 0.78 & 2.44 \\
\hline $07 \mathrm{E}(68)$ & 27 & 39.7 & 0.91 & 6.16 \\
\hline 07L (79) & 20 & 25.3 & 0.89 & 4.35 \\
\hline 08E (78) & 25 & 32.1 & 0.89 & 5.51 \\
\hline $\begin{array}{l}\chi^{2} \text { for } \\
\text { homogeneity }\end{array}$ & & $32.97 * \mathrm{e}$ & 0.17 & 5.97 \\
\hline
\end{tabular}

a 99L, the later cropping season (July-December 1999); 00E, the earlier cropping season (March-July 2000), and so on.

b Race diversity index within each population from season to season. Higher values (maximum of 1) indicate greater diversity.

$\mathrm{c}$ Margalef index of race richness within each population from season to season. The higher the value, the richer the race of a given population.

${ }^{\mathrm{d}} \chi^{2}$ test for homogeneity applied to determine whether a given parameter varied across the populations.

e Asterisks indicate values of the parameter differed significantly $(P<0.05$, $d f=17)$.
(ZC13 and ZC15) occurred in all 18 seasons (Table 3; Supplementary Table S1). Similarly, a study of $P$. oryzae populations in the United States over the period 1959 to 2015 found that just three out of 39 races occurred every year (Wang et al. 2017). Rather differently, a survey of HLJ $P$. oryzae populations from 2006 to 2015 revealed that nine out of 30 races (five indica and four japonica types) could be classified as common (Zhang et al. 2019). The total population common race isolate frequency $\left(f_{t p c r i}\right)$ parameter also differed markedly between GD and HLJ (7.4 to $51.5 \%$ in the former [Table 3] but 51.0 to $93.8 \%$ in the latter [Zhang et al. 2019]). It clearly indicated that there was a higher diversity in $P$. oryzae populations in GD and much less in HLJ. On the other hand, nine indica type races (ZB1, ZB5, ZB6, ZB7, ZB13, ZB15, ZC5, ZC13, and ZC15) and one japonica type race ( $\mathrm{ZG} 1)$ were recognized as the dominant races in GD from 1999 to 2008 (Table 4; Supplementary Table S2). It was consistent with the identification of four indica type races (ZB1,

Table 2. Specific races of Pyricularia oryzae in Guangdong province over the period 1999-2008

\begin{tabular}{|c|c|c|c|c|c|}
\hline \multirow{2}{*}{$\begin{array}{l}\text { Population } \\
(\Sigma)^{\mathbf{a}}\end{array}$} & \multirow{2}{*}{$\begin{array}{l}\text { Population-specific race (psr) } \\
\text { (no. of isolates) })^{\mathbf{b}}\end{array}$} & \multicolumn{2}{|c|}{ Race } & \multicolumn{2}{|c|}{ Isolate } \\
\hline & & No. & $f_{p s r}$ & No. & $f_{t p s r i}$ \\
\hline 00E (59) & ZD3 (1) & 1 & 10.0 & 1 & 1.7 \\
\hline $01 \mathrm{~L}(47)$ & ZA9 (1) & 1 & 5.9 & 1 & 2.1 \\
\hline 03E (80) & ZB17 (1) & 1 & 4.8 & 1 & 1.3 \\
\hline 04L (78) & ZA29 (1), ZA63 (1) & 2 & 10.5 & 2 & 2.6 \\
\hline 05E (58) & ZA21 (1) & 1 & 7.7 & 1 & 1.7 \\
\hline 07E (68) & $\begin{array}{l}\mathrm{ZB} 2 \text { (2), ZC8 (1), ZC14 (2), ZD8 } \\
\text { (2) }\end{array}$ & 4 & 14.8 & 7 & 10.3 \\
\hline 08E (78) & $\begin{array}{l}\text { ZA13 (1), ZA31 (1), ZB8 (1), } \\
\text { ZB30 (1) }\end{array}$ & 4 & 16.0 & 4 & 5.1 \\
\hline \multicolumn{2}{|c|}{$\chi^{2}$ for homogeneity ${ }^{c}$} & & 10.89 & & $17.75^{* \mathrm{~d}}$ \\
\hline
\end{tabular}

${ }^{a}$ Out of 18 populations, seven were identified by their specific races.

${ }^{\mathrm{b}}$ Races present in an individual population, which were ordered according to their alphanumeric code.

${ }^{c} \chi^{2}$ test for homogeneity applied to determine whether a given parameter varied across the populations.

$\mathrm{d}$ Asterisks indicate values of the parameter differed significantly $(P<0.01$, $d f=6)$.

Table 3. Common races of Pyricularia oryzae in Guangdong province over the period 1999-2008

\begin{tabular}{|c|c|c|c|c|c|c|}
\hline \multirow[b]{2}{*}{ 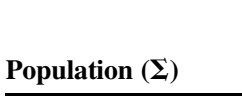 } & \multicolumn{2}{|c|}{$\mathrm{ZC13}^{\mathrm{a}}$} & \multicolumn{2}{|c|}{ ZC15 } & \multirow[b]{2}{*}{$f_{p c r}$} & \multirow[b]{2}{*}{$f_{t p c r i}$} \\
\hline & No. & $f_{\text {pcri-1 }}$ & No. & $f_{\text {pcri-2 }}$ & & \\
\hline 99L (51) & 2 & 3.9 & 9 & 17.6 & 20.0 & 21.6 \\
\hline O0E (59) & 1 & 1.7 & 18 & 30.5 & 20.0 & 32.2 \\
\hline 00L (97) & 17 & 17.5 & 33 & 34.0 & 11.8 & 51.5 \\
\hline $01 \mathrm{E}(57)$ & 6 & 10.5 & 16 & 28.1 & 18.2 & 38.6 \\
\hline $01 \mathrm{~L}(47)$ & 9 & 19.1 & 2 & 4.3 & 11.8 & 23.4 \\
\hline 02E (85) & 10 & 11.8 & 3 & 3.5 & 13.3 & 15.3 \\
\hline 02L (75) & 18 & 24.0 & 17 & 22.7 & 18.2 & 46.7 \\
\hline 03E (80) & 17 & 21.3 & 6 & 7.5 & 9.5 & 28.8 \\
\hline 03L (81) & 9 & 11.1 & 21 & 25.9 & 11.1 & 37.0 \\
\hline 04E (78) & 10 & 12.8 & 1 & 1.3 & 12.5 & 14.1 \\
\hline 04L (78) & 7 & 9.0 & 8 & 10.3 & 10.5 & 19.2 \\
\hline 05E (58) & 4 & 6.9 & 2 & 3.4 & 15.4 & 10.3 \\
\hline 05L (57) & 6 & 10.5 & 5 & 8.8 & 14.3 & 19.3 \\
\hline $06 \mathrm{E}(60)$ & 4 & 6.7 & 2 & 3.3 & 14.3 & 10.0 \\
\hline 06L (60) & 12 & 20.0 & 1 & 1.7 & 18.2 & 21.7 \\
\hline 07E (68) & 3 & 4.4 & 2 & 2.9 & 7.4 & 7.4 \\
\hline 07L (79) & 10 & 12.7 & 8 & 10.1 & 10.0 & 22.8 \\
\hline 08E (78) & 5 & 6.4 & 1 & 1.3 & 8.0 & 7.7 \\
\hline$\chi^{2}$ for homogeneity ${ }^{b}$ & & $60.23 * \mathrm{c}$ & & $179.05^{*}$ & 20.09 & $122.06^{*}$ \\
\hline
\end{tabular}

a Race present in all 18 populations.

${ }^{\mathrm{b}} \chi^{2}$ test for homogeneity applied to determine whether a given parameter varied across the populations.

${ }^{c}$ Asterisks indicate values of the parameter differed significantly $(P<0.001$, $d f=17)$. 
ZB5, ZB13, and ZC13) as dominant in GD between 1991 and 1994 (Yang et al. 1995) and three indica type races (ZB13, ZC13, and ZC15) and one japonica (ZG1) from 1995 to 1997 (Pan et al.

Table 4. Dominant races of Pyricularia oryzae in Guangdong province over the period 1999-2008

\begin{tabular}{|c|c|c|c|c|c|}
\hline \multirow[b]{2}{*}{ Population $(\Sigma)$} & \multicolumn{4}{|c|}{ Top 2 races and their frequencies ${ }^{a}$} & \multirow[b]{2}{*}{ Top $2 f_{t t 2 r i}$} \\
\hline & 1st (no.) & $f_{t 2 r i-1}$ & 2nd (no.) & $f_{t 2 r i-2}$ & \\
\hline 99L (51) & ZG1 (29) & 56.9 & ZC15 (9) & 17.6 & 74.5 \\
\hline O0E (59) & ZC15 (18) & 30.5 & ZB15 (11) & 18.6 & 49.2 \\
\hline 00L (97) & ZC15 (33) & 34.0 & ZC13 (17) & 17.5 & 51.5 \\
\hline $01 \mathrm{E}(57)$ & ZC15 (16) & 28.1 & ZB15 (11) & 19.3 & 47.4 \\
\hline $01 \mathrm{~L}(47)$ & ZC13 (9) & 19.1 & ZB13 (5) & 10.6 & 29.8 \\
\hline 02E (85) & ZB5 (17) & 20.0 & ZB7 (11) & 12.9 & 32.9 \\
\hline 02L (75) & ZC13 (18) & 24.0 & ZC15 (17) & 22.7 & 46.7 \\
\hline 03E (80) & ZC13 (17) & 21.3 & ZB5 (13) & 16.3 & 37.5 \\
\hline 03L (81) & ZC15 (21) & 25.9 & ZG1 (10) & 12.3 & 38.3 \\
\hline 04E (78) & ZB5 (41) & 52.6 & ZC13 (10) & 12.8 & 65.4 \\
\hline 04L (78) & ZC5 (15) & 19.2 & ZB5 (11) & 14.1 & 33.3 \\
\hline 05E (58) & ZB5 (35) & 60.3 & $\mathrm{ZC} 13(4)$ & 6.9 & 67.2 \\
\hline 05L (57) & ZB5 (18) & 31.6 & ZC5 (14) & 24.6 & 56.1 \\
\hline $06 \mathrm{E}(60)$ & ZB5 (26) & 43.3 & ZB7 (5) & 8.3 & 51.7 \\
\hline 06L (60) & ZB5 (22) & 36.7 & ZC5 (13) & 21.7 & 58.3 \\
\hline 07E (68) & ZB5 (18) & 26.5 & ZB6 (6) & 8.8 & 35.3 \\
\hline 07L (79) & ZB5 (20) & 25.3 & $\mathrm{ZC} 13(10)$ & 12.7 & 38.0 \\
\hline 08E (78) & ZB5 (23) & 29.5 & ZB6 (11) & 14.1 & 43.6 \\
\hline \multicolumn{2}{|c|}{$\chi^{2}$ for homogeneity ${ }^{b}$} & $85.68^{* *}$ & & $28.75^{*}$ & $59.51 * *$ \\
\hline
\end{tabular}

a Top 2 races within each population, and in the cases of 2 parallel second races present [Pop-00E (ZB15, ZG1), Pop-05E (ZB1, ZC13), and Pop07L (ZC5, ZC13)], only 1 listed in the table was accounted (also see Supplementary Table S1 and S4). *, values of the parameter differed significantly at $P=0.05(d f=17)$; **, values of the parameter differed significantly at $P=0.001(d f=17)$.

${ }^{b} \chi^{2}$ test for homogeneity applied to determine whether a given parameter varied across the populations.
1998). Meanwhile, in HLJ, four japonica type races (ZD1, ZD3, ZD5, and ZE1) were classed as dominant from 2006 to 2015 (Zhang et al. 2019). Together, the distinct race structures of $P$. oryzae populations between GD and HLJ indicated that there was a certain difference in both number and content of resistance genotypes that have been used in the respective regions (Zhang et al. 2017, 2019).

Clearer information on race structure-illustrated resistance profiles of differential cultivars is crucial to plant breeders to create greater cultivars with promising resistance (Fukuta et al. 2019; Liu et al. 2017; Zhang et al. 2017, 2019). The CDCs were established based on screening of 212 rice cultivars with 1,739 P. oryzae isolates that were selected across China (All China Corporation of Research on Physiological Races of Pyricularia oryzae 1980). Because the CDC set consists of both indica and japonica cultivars in which $>15$ specific resistance genes, which were widely used in the Chinese rice breeding programs, were involved (Zhang et al. 2017; Q. Pan et al., unpublished data), it was more suitable for Chinese $P$. oryzae populations, compared with the Japanese differential cultivars and even near isogenic differentials (Chen et al. 2001; Ling et al. 2004; Xing et al. 2017; Yang et al. 2004; Zhang et al. 2017, 2019). When the host resistance of the CDC panel was characterized with $P$. oryzae populations sampled in HLJ from 2006 to 2015, the three indica type members (Tetep, Zhenlong 13, and Sifeng 43) all exhibited a higher level of resistance than the four japonica type members (Dongnong 363, Kanto 51, Heijiang 18, and LTH) (Zhang et al. 2019). Here, rather differently, dynamics of resistance with the CDCs were divided into three types, irrespective of indica and japonica type entries: Tetep and Kanto 51 expressed the highest and most stable level of resistance, whereas Sifeng 43 and LTH had lower and dynamic resistance, and the other three, Zhenlong 13, Dongnong 363, and Heijiang 18, exhibited intermediate and seasonally dynamic resistance across the 18 seasons (Fig. 1; Supplementary Table S4). This different outcome undoubtedly reflects the distinctness of race structures of the $P$. oryzae populations present in these two geographically well separated regions of China (the distance from Harbin [HLJ] to

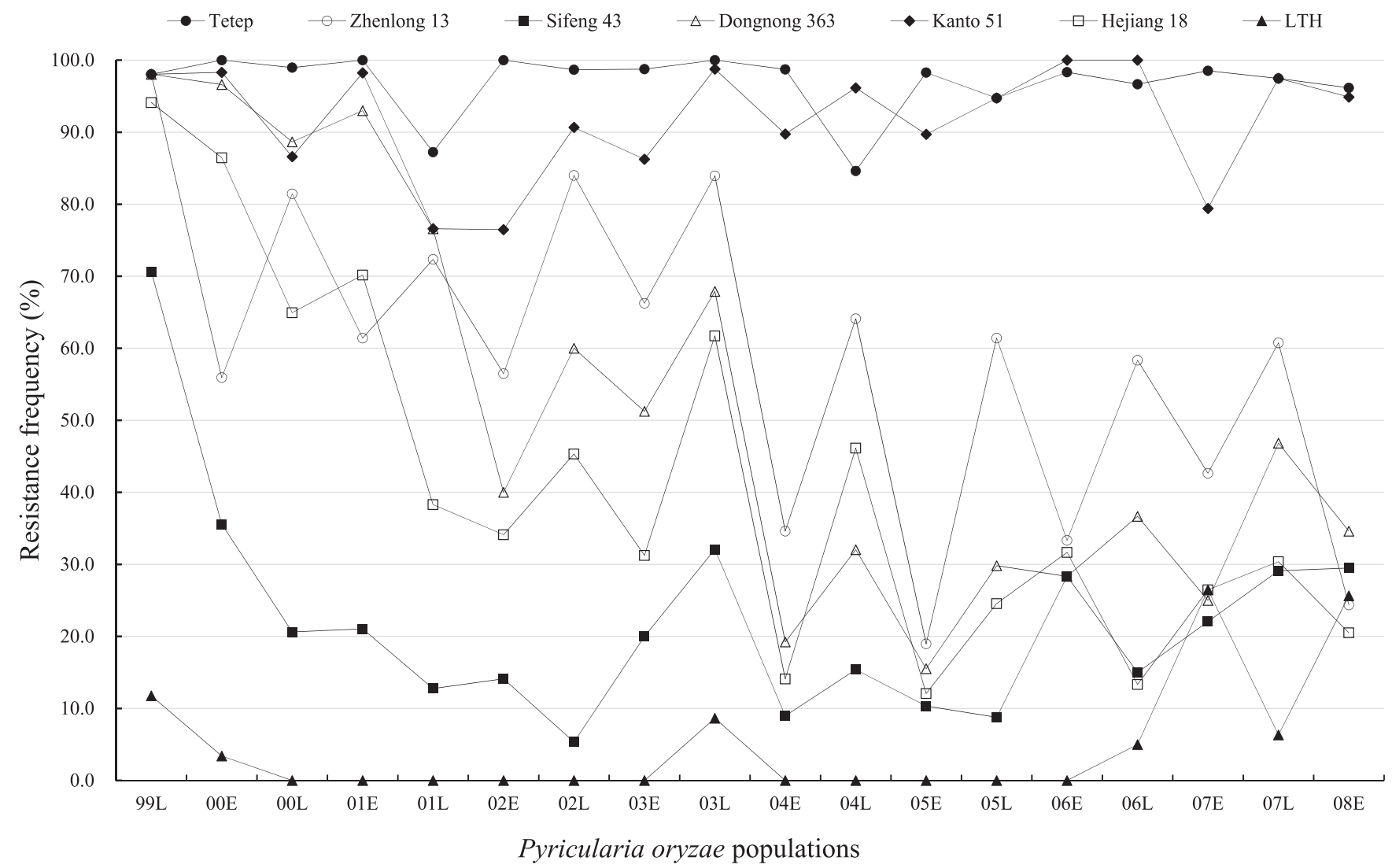

Fig. 1. Temporal variation in the resistance frequency of each Chinese differential cultivar challenged by Guangdong populations of Pyricularia oryzae. 99L, the later season (July-December 1999); 00E, the earlier season (March-July) 2000, and so on. 
Guangzhou [GD] is nearly 3,000 km). A reasonable point derived from both studies is that host resistance genes originating in both indica and japonica type donors have been widely used for rice improvement in GD, and the breeding effort in HLJ has focused much more strongly on japonica type donors, particularly on their resistance genes, Pia, Pii, and Pik (Zhang et al. 2017, 2019).

It was noteworthy that the performance of the universal susceptible cultivar, LTH, was abnormal in both 07E-Pop and 08E-Pop, because the resistance frequencies in both populations reached $>20 \%$ in the current study (Fig. 1). This effect might be because of an additional resistance gene, Pik-l (Singh et al. 2015), which was effective against some isolates in both populations. That is, LTH still carries resistance genes depending on isolates being tested. Another insight derived from this phenomenon was that it might be relative to a certain change in deployment of rice cultivars in such populations.

It was interesting that Zhenlong 13, Dongnong 363, and Hejiang 18 each had a lower resistance frequency in the earlier cropping season than in the later one (Fig. 1). The host genotypes, particularly the common resistance gene, Pia, being carried by the three cultivars, might be responsible to the environmental factors in the local ecosystem (Zhang et al. 2017, 2019). On the pathogen side, the implication of this observation is that $P$. oryzae populations are able adapt rapidly to the different environments between the two seasons. An analysis of the race structure of $P$. oryzae populations collected in GD in 2000 and 2001 has shown the presence of two lineages, one derived from plants sampled during the earlier cropping season and the other during the later cropping season (Hu et al. 2002; Pan et al. 2003b). However, it is unclear what environmental factors could be responsible for shaping this apparent seasonal effect on the pathogen's race structure. Additional studies are needed to identify the environmental factors.

\section{Literature Cited}

All China Corporation of Research on Physiological Races of Pyricularia oryzae. 1980. Research on physiological races of rice blast fungus in China. Acta Phytopathol. Sin. 10:71-82.

Atkins, J., Robert, A., Adair, C., Goto, K., Kozaka, T., Yanagida, R., Yamada, M., and Matsumoto, S. 1967. An international set of rice varieties for differentiating races of Piricularia pryzae. Phytopathology 57:297-301.

Chen, H., Chen, B., Zhang, D., Xie, Y., and Zhang, Q. 2001. Pathotypes of Pyricularia grisea in rice fields of central and southern China. Plant Dis. 85: 843-850.

Cheng, S. 2010. Retrospect and prospect of rice breeding in China. Pages 1-23 in: Rice Breeding and Cultivar Genealogies in China During 1986 to 2005. J. Wan, ed. China Agr. Press, Beijing, China.

Correa-Victoria, F., and Zeigler, R. 1993. Pathogenic variability in Pyricularia grisea at a rice blat "hot spot" breeding site in Eastern Colombia. Plant Dis. 77:1029-1035

Couch, B. C., and Kohn, L. M. 2002. A multilocus gene genealogy concordant with host preference indicates segregation of a new species, Magnaporthe oryzae, from M. grisea. Mycologia 94:683-693.

Dorrance, A., Kurle, J., Robertson, A., Bradley, C., Giesler, L., Wise, K., and Concibido, V. 2016. Pathotype diversity of Phytophthora sojae in eleven states in the United States. Plant Dis. 100:1429-1437.

Feng, C., Saito, K., Liu, B., Manley, A., Kammeijier, K., Mauzey, S., Koike, S., and Correll, J. 2018. New races and novel strains of the spinach downy mildew pathogen Peronospora effuse. Plant Dis. 102:613-618.

Fernando, D., Zhang, X., Selin, C., Zou, Z., Liban, S., McLaren, D., Kubinec, A., Parks, P., Rashid, H., Pamathilake, R., Rong, L., Yang, C., Gnanesh, B., and Huang, S. 2018. A six-year investigation of the dynamics of avirulence allele profiles, blackleg incidence, and mating type alleles of Leptosphaeria maculans populations associated with canola crops in Manitoba, Canada. Plant Dis. 102:790-798.

Fukuta, Y., Telebanco-Yanoria, M., Hayashi, N., Yanagihara, S., Machungo, C., and Makihara, D. 2019. Pathogenicities of rice blast (Pyricularia oryzae Cavara) isolates from Kenya. Plant Dis. 103:3181-3188.

Gao, X., Jin, Z., Li, Z., Zhang, X., Han, B., and Zhang, Z. 2017. Identification of rice blast pathogen races in Heilongjiang in 2015. China Rice 23:50-53.

Hu, Z., Chen, J., Wang, L., Qiu, Q., and Pan, Q. 2002. Molecular genetic studies on the rice blast fungus population. II. Temporal and spatial characterization of genetic structure of the fungus population in 2000 in Guangdong province. Mycosystema 21:203-209.

Huo, C., Liu, Z., and Zhou, L. 1980. Studies on physiological races of Pyricularia oryzae in Guangdong province. Guangdong Agr. Sci. 3:41-45.
Jindal, K., Tenuta, A., Woldemariam, T., Zhu, X., Hooker, D., and Reid, L. 2019. Occurrence and distribution of physiological races of Exserohilum turcicum in Ontario, Canada. Plant Dis. 103:1450-1457.

Lai, Z., Huang, J., Xu, Q., and Zou, S. 1998. Dynamics and regional distribution of races of the rice blast pathogen in Guangdong province. Plant Prot. Tech. Ext. Supplementary Issue: 7-10.

Lei, C., Zhang, G., Cheng, Z., Ma, J., Wang, J., Xin, A., Chen, P., Xiao, J., Zhang, X., Liu, Y., Guo, X., Wang, J., Zhai, H., and Wan, J. 2011. Pathogenic races and virulence gene structure of Magnaporthe oryzae population and rice breeding strategy for blast resistance in Heilongjiang province. Acta Agron. Sin. 37:18-27.

Li, H., and Zheng, G. 1989. Studies on dynamics of races of Magnaporthe oryzae populations collected in Heilongjiang province, China. Plant Prot. 15:2-4

Li, T., Wen, J., Zhang, Y., Correll, J., Wang, L., and Pan, Q. 2018. Reconstruction of an SSR-based Magnaporthe oryzae physical map to locate avirulent gene AvrPi12. BMC Microbiol. 18:47.

Ling, Z., Lei, C., and Wang, J. 2004. Review and prospect for study of physiological races on rice blast fungus (Pyricularia grisea). Sci. Agr. Sin. $37: 1849-1859$

Liu, T., Wan, A., Liu, D., and Chen, X. 2017. Changes of races and virulence genes in Puccinia striiformis $\mathrm{f}$. sp. tritici, the wheat stripe rust pathogen, in the United States from 1968 to 2009. Plant Dis. 101:1522-1532.

Mei, F., Wu, X., Yao, C., and Li, L. 1988. Rice cropping regionalization in China. Pages 1-48 in: Regionalization of Rice Cropping in China. China National Rice Research Institute, ed. Zhejiang Sci.-Tech. Publishing House, Hangzhou, China.

Nguyet, N., Long, H., Ngoc, N., Nhai, N., Thuy, N., Hayashi, N., and Fukuta, Y 2020. Diversity and distribution of rice blast (Pyricularia oryzae Cavara) races in Vietnam. Plant Dis. 104:381-387.

Paczos-Grzęda, E., and Sowa, S. 2019. Virulence structure and diversity of Puccinia cronata f. sp. avenae P. Syd. \& Syd. in Poland during 2013 to 2015. Plant Dis. 103:1559-1564.

Pan, Q., Hu, Z., Tanisaka, T., and Wang, L. 2003a. Fine mapping of the blast resistance gene $\mathrm{Pi15}$, linked to $\mathrm{Pii}$, on rice chromosome 9. J. Integr. Plant Biol. 45:871-877

Pan, Q., Wang, L., Chen, J., Luo, Q., and Hu, Z. 2003b. Molecular genetic studies on the rice blast fungus population. III. Genetic structure of the fungus population in 2001 in Chinese Guangdong and two hypotheses proposed. Mycosystema 22:62-68.

Pan, Q., Wang, L., Ikehashi, H., and Tanisaka, T. 1996. Identification of a new blast resistance gene in the indica rice cultivar Kasalath using Japanese differential cultivars and isozyme markers. Phytopathology 86:1071-1075.

Pan, R., Kang, B., Huang, J., and Xu, Q. 1998. The distribution of physiological races of rice blast fungus Magnaporthe grisea in Guangdong province. Plant Prot. Tech. Ext. 18:3-5

Shang, S., Li, M., Piao, M., Zhang, X., Zhang, Y., Yu, J., and Liu, H. 1996 Identification of the physiological races of Piricularia oryzae (Magnaporthe oryzae) in Heilongjiang province. Plant Prot. 22:11-14.

Shi, M., Liu, Z., Chen, Y., Tian, A., Yao, L., Ren, C., Miao, Q., Yu, H., and Cheng, Z. 2015. Population diversity of Magnaporthe oryzae of Jiangsu and Liaoning japonica rice area. Acta Phytopathol. Sin. 45:158-166.

Singh, W., Kapila, R., Sharma, T., and Rathou, R. 2015. Genetic and physical mapping of a new allele of Pik locus from japonica rice 'Lijiangxintuanheigu'. Euphytica 205:889-901

Wang, X., Jia, Y., Wamishe, Y., Jia, M., and Valent, B. 2017. Dynamic changes in the rice blast population in the USA over six decades. Mol. Plant-Microbe Interac. 30:803-812.

Wu, W., Wang, L., He, Y., and Pan, Q. 2004. Molecular genetic studies on the rice blast fungus population: comparison of genetic pathotypic structures of two rice blast fungus populations originated from Guangdong and Jiangsu provinces, China. Sci. Agric. Sinica. 37:1628-1635.

Xing, J., Jia, Y., Peng, Z., Shi, Y., He, Q., Shu, F., Zhang, W., Zhang, Z., and Deng, H. 2017. Characterization of molecular identity and pathogenicity of rice blast fungus in Hunan province of China. Plant Dis. 101:557-561.

$\mathrm{Xu}, \mathrm{Z}$. 1988. Regionalization of rice cropping in Guangdong province. Pages 53-56 in: Regionalization of Rice Cropping in China. China National Rice Research Institute ed. Zhejiang Sci.-Tech. Publishing House, Hangzhou, China.

Yang, Q., Zhu, X., and Sun, Y. 1995. Distribution and dynamics of races of Pyricularia oryzae in Guangdong province. Guangdong Agr. Sci. 4:39-41.

Yang, X., Wang, L., He, Y., and Pan, Q. 2004. Molecular genetic studies on the rice blast fungus population: Dynamics of genetic and pathotypic structures of three rice blast fungus populations originated from Guangdong province in 2000 to 2002. Sci. Agric. Sinca 37:1468-1473.

Zhang, Y., Wang, J., Jin, X., Yao, Y., Correll, J., Wang, L., and Pan, Q. 2019 Dynamics of race structure of the rice blast pathogen populations in Heilongjiang province, China from 2006 through 2015. Plant Dis. 103: 2759-2763

Zhang, Y., Zhu, Q., Yao, Y., Zhao, Z., Correll, J., Wang, L., and Pan, Q. 2017. The race structure of the rice blast pathogen across southern and northeastern China Rice 10:46. 